\title{
Erratum to: Contemporary Reviews of Mine Water Studies in Europe, Part 2
}

\author{
Christian Wolkersdorfer • Rob Bowell • Ingar F. Walder · Steinar Nilssen • Marja Liisa Räisänen • \\ Päivi Heikkinen • Kari Pulkkinen • Kirsti Korkka-Niemi • Veli-Pekka Salonen • Georgia Destouni • \\ Andrea Hasche $\cdot$ Andrzej J. Witkowski • A. Blachère $\cdot$ S. Morel $\cdot$ D. Lefort $\cdot$ Sanda Midžić $\cdot$ \\ Irem Silajdžić • R. H. Coulton · K. P. Williams • Ben Rees $\cdot$ Kevin B. Hallberg • D. Barrie Johnson
}

Published online: 19 June 2012

(C) Springer-Verlag 2012

Erratum to: Mine Water and the Environment (2005)

\section{4:2-37}

DOI 10.1007/s10230-005-0068-0

The names of some of the authors of this section were inadvertently omitted in the original publication. We regret this omission and provide all of the authors' names here:

The online version of the original article can be found under doi:10.1007/s10230-005-0068-0.

\section{Wolkersdorfer $(\bowtie)$}

Technische Universität Bergakademie Freiberg,

Lehrstuhl für Hydrogeologie, Gustav-Zeuner-Str. 12,

09596 Freiberg/Sachsen, Germany

e-mail: christian@wolkersdorfer.info

R. Bowell

SRK Consulting, Windsor Court, 1 Windsor Place,

Cardiff CF10 3BX, Wales, UK

I. F. Walder

Kjeøy Research and Education Center, Kjeøy,

8581 Vestbygd, Norway

\section{S. Nilssen}

Norwegian Commission of Mines, Postbox 3021 Lade,

7441 Trondheim, Norway

\section{L. Räisänen}

Geological Survey of Finland/University of Kuopio,

PO Box 1237, 70211 Kuopio, Finland

P. Heikkinen

Geological Survey of Finland, PO Box 96,

02151 Espoo, Finland
Wolkersdorfer, Christian; Bowell, Rob; Walder, Ingar F.; Nilssen, Steinar; Räisänen, MarjaLiisa; Heikkinen, Päivi; Pulkkinen, Kari; Korkka-Niemi, Kirsti; Salonen, Veli-
K. Pulkkinen

Outokumpu Mining Oy, Hitura Mine, Kummuntie 8, 85660 Ainastalo, Finland

K. Korkka-Niemi

Department of Geology, University of Turku, 20500 Turku, Finland

\section{V.-P. Salonen}

Department of Geology, University of Helsinki,

PO Box 64, 00014 Helsinki, Finland

\section{G. Destouni}

Department of Physical Geography and Quaternary Geology, Stockholm University, 10691 Stockholm, Sweden

A. Hasche

TU Bergakademie Freiberg, Gustav-Zeuner-Straße 12, 09596 Freiberg, Germany

A. J. Witkowski

Department of Hydrogeology and Engineering Geology,

University of Silesia, Będzińska 60, 41-200 Sosnowiec, Poland 
Pekka; Destouni, Georgia; Hasche, Andrea; Witkowski, Andrzej J.; Blachère, A.; Morel, S.; Lefort, D.; Midžić,
Sanda; Silajdžić, Irem; Coulton, R. H.; Williams, K. P.; Rees, Ben; Hallberg, Kevin B.; Johnson, D. Barrie
A. Blachère $\cdot$ S. Morel $\cdot$ D. Lefort

Bureau d'etudes CESAME, Z.A. du Parc, Secteur Gampille, 42490 Fraisses, France

S. Midžić · I. Silajdžić

Hydro-Engineering Institute Sarajevo, Stjepana Tomića 1,

71000 Sarajevo, Bosnia and Herzegovina

R. H. Coulton

Unipure Europe Ltd, Unipure House, Wonastow Road,

Monmouth NP25 5JA, UK
K. P. Williams

School of Engineering, Cardiff University, PO Box 925, Cardiff CF24 OYF, UK

B. Rees

SRK Consulting, Windsor Court, 1-3 Windsor Place, Cardiff, CF10 3BX, UK

K. B. Hallberg · D. B. Johnson

School of Biological Sciences, University of Wales, Bangor, Memorial Bldg, Deiniol Rd, Bangor LL57 2UW, UK 